\title{
THE RANGES OF CERTAIN CONVOLUTION OPERATORS
}

\author{
EDWIN HEWITT
}

\section{Introduction.}

A typical result of this note is that $\mathfrak{L}_{1}(G) * \mathfrak{L}_{p}(G)=\mathfrak{L}_{p}(G), 1<p<\infty$, where $G$ is an arbitrary locally compact group. Several similar equalities are obtained: for the exact results, see (3.2)-(3.6). Our basic idea is borrowed from Paul J. Cohen's interesting paper [1]. (N. Th. Varopoulos [5] has also made use of Cohen's construction to show that every positive functional on $\mathfrak{L}_{1}(G)$ is continuous.) We have benefited from conversations with Irving L. Glicksberg, Andrzej Hulanicki, and Kenneth A. Ross.

While our main concern is with algebras of measures operating by convolution on the Banach spaces $\mathfrak{Q}_{p}(G)$, our main "factorization" theorem is most economically stated and proved for Banach algebras operating on Banach spaces. The needed definitions, statements, and proofs appear in Section 2, the applications to convolution operators in Section 3. All terms and notation not explained here are as in the monograph [2].

\section{The general factorization theorem.}

(2.1) Definition. Let $A$ be a complex or real Banach algebra with elements $\mu, v, \sigma, \tau, \ldots$ and norm $\|\cdot\|$. Let $L$ be a complex or real Banach space with elements $x, y, z, \ldots$ and norm \|\|$\cdot \|$. We suppose that $A$ is real if $L$ is real. We suppose that there is a mapping of $A \times L$ into $L$, and we write the image of $(\mu, x)$ as $\mu \bullet x$. We suppose that this mapping has the following properties:

(i) $(\mu+\nu) \cdot x=(\mu \cdot x)+(\nu \cdot x)=\mu \cdot x+\nu \cdot x$;

(ii) $(t \mu) \cdot x=t(\mu \cdot x)=\mu \cdot(t x)$ for all $t$ in the scalar field of $L$;

(iii) $(\mu \nu) \cdot x=\mu \cdot(\nu \cdot x)$;

(iv) $\|\mu \mu \cdot x\|\|\leqq c\| \mu\|\cdot\| \mid x\|\|$, where $c$ is a real constant $\geqq 1$.

(v) We also suppose that for every finite set $\left\{\mu_{1}, \ldots, \mu_{m}\right\} \subset A$, every

Received March 10, 1965.

Research supported in part by the U.S. National Science Foundation. 
$x \in L$, and every positive real number $a$, we can choose a single element $\nu \in A$ such that $\|v\| \leqq d$ (where $d$ is a positive constant) and $\left\|\nu \mu_{j}-\mu_{j}\right\|<a$, $j=1,2, \ldots, m$, and $\|\nu \cdot x-x \mid\|<a$.

Thus $A$ has a bounded approximate left unit, which also acts restrictedly like an approximate left unit under •

(2.2) Construction. Let $A_{\varepsilon}$ be the algebra obtained from $A$ by adjoining a unit $\varepsilon$, and with the customary norm $\|\mu+t \varepsilon\|=\|\mu\|+|t|$. For $\mu+t \varepsilon \in A_{\varepsilon}$ and $x \in L$, let $(\mu+t \varepsilon) \bullet x=\mu \bullet x+t x$. Properties (2.1.i)-(2.1.iii) are evident for the extended operation $\bullet$, and (2.1.iv) holds because $c \geqq 1$.

(2.3) Lemma. Let $d$ be a real number $\geqq 1$. For $\mu \in A$ such that $\|\mu\| \leqq d$, let $\varphi(\mu)$ be the element of $A_{8}$ defined by

(i) $\varphi(\mu)=\left(\frac{2 d+1}{2 d}\right)\left[\varepsilon+\sum_{k=1}^{\infty}(-1)^{k}(2 d)^{-k} \mu^{k}\right]$.

Then we have:

(ii) $\varphi(\mu)=\left[\frac{2 d}{2 d+1} \varepsilon+\frac{1}{2 d+1} \mu\right]^{-1}$;

(iii) $\left\|\left|\varphi(\mu) \cdot x-x\|\| \leqq\left(2+d^{-1}\right) c\right|\right\| \mu \cdot x-x\|\|$;

and

(iv) $\frac{2}{3}+d^{-1} \leqq\|\varphi(\mu)\| \leqq 2+d^{-1}$.

Proof. The infinite series $\sum_{k=1}^{\infty}(-1)^{k}(2 d)^{-k} \mu^{k}$ obviously converges in $A$, as $A$ is complete and $\left\|\mu^{k}\right\| \leqq d^{k}$. Since

$$
\left(\varepsilon+(2 d)^{-1} \mu\right)\left(\varepsilon+\sum_{k=1}^{n}(-1)^{k}(2 d)^{-k} \mu^{k}\right)=\varepsilon+(-1)^{n}(2 d)^{-(n+1)} \mu^{n+1},
$$

(ii) holds. Now let $x$ be an arbitrary element of $L$. The definition (i) and a trivial computation show that

so that

$$
\varphi(\mu) \bullet x-x=\frac{2 d+1}{2 d} \sum_{k=1}^{\infty}(-1)^{k}(2 d)^{-k}\left(\mu^{k} \cdot x-x\right),
$$

$$
\left\|\varphi(\mu) \cdot x-x\left|\left\|\leqq \frac{2 d+1}{2 d} \sum_{k=1}^{\infty}(2 d)^{-k} \mid\right\| \mu^{k} \cdot x-x\|\| .\right.\right.
$$

For each $k$, we have 


$$
\begin{aligned}
\left\|\mu^{k} \cdot x-x\right\| \| & \leqq \sum_{l=1}^{k}\left\|\mu^{l} \cdot x-\mu^{l-1} \cdot x\right\| \| \\
& \leqq \sum_{l=1}^{k} c\|\mu\|^{l-1}\|\mu \cdot x-x\| \| \\
& \leqq c k d^{k}\|\mu \cdot x-x\| \| .
\end{aligned}
$$

Substitution of (2) in (1) yields

$$
\begin{aligned}
\|\varphi(\mu) \cdot x-x \mid\| & \leqq\left(\frac{2 d+1}{2 d}\right)\left(\sum_{k=1}^{\infty} c k 2^{-k}\right)\|\| \mu \cdot x-x\|\| \\
& =c\left(2+d^{-1}\right)\|\mid \mu \cdot x-x\| \| \\
& \leqq 3 c\|\mid\| \cdot x-x\|\|,
\end{aligned}
$$

which is (iii). The inequalities (iv) follow at once from (i) and the relation

$$
\varepsilon=\varphi(\mu)\left(\frac{2 d}{2 d+1} \varepsilon+\frac{1}{2 d+1} \mu\right) .
$$

(2.4) Lemma. Let $a$ be an arbitrary positive number and $z$ an arbitrary element of $L$. Let $d$ be an upper bound on the norms of approximate units in $A$ as in (2.1.v). There is a sequence $\left(\mu_{n}\right)_{n=1}^{\infty}$ of elements of $A$ with norms $\leqq d$ and having the following properties. Write

(i) $\sigma_{n}=\sum_{k=1}^{n}(2 d)^{k-1}(2 d+1)^{-k} \mu_{k}+(2 d)^{n}(2 d+1)^{-n} \varepsilon$ for $n=1,2,3, \ldots$, and $\sigma_{0}=\varepsilon$.

Then $\sigma_{n}^{-1}$ exists in $A_{\varepsilon}$ and

(ii) ||$\sigma_{n}^{-1} \bullet z-\sigma_{n-1}^{-1} \bullet z \mid \| \leqq 2^{-n} a$ for $n=1,2,3, \ldots$

Proof. Using (2.1.v), choose as $\mu_{1}$ any element of $A$ such that $\left\|\mu_{1}\right\| \leqq d$ and $\left\|\mu_{1} \cdot z-z \mid\right\| \leqq\left(2 c\left(2+d^{-1}\right)\right)^{-1} a$. Then

$$
\sigma_{1}=\frac{1}{2 d+1} \mu_{1}+\frac{2 d}{(2 d+1)} \varepsilon .
$$

Thus $\sigma_{1}^{-1}$ is the $\varphi\left(\mu_{1}\right)$ of Lemma (2.3), and (2.3.iii) implies that

$$
\left\|\left|\sigma_{1}^{-1} \bullet z-\sigma_{0}^{-1} \bullet z\right|\right\| \leqq\left(2+d^{-1}\right) c\left|\left\|\mu_{1} \bullet z-z \mid\right\| \leqq 2^{-1} a .\right.
$$

Thus (ii) holds for $n=1$.

We define $\mu_{2}, \mu_{3}, \ldots$ by finite induction. Suppose that $\mu_{1}, \mu_{2}, \ldots, \mu_{m}$ have been defined so that $\sigma_{n}^{-1}$ exists in $A_{8}$ and satisfies (ii) for $n=1,2$, $\ldots, m$. To see how $\mu_{m+1}$ should be chosen, consider any element $\mu^{\prime}$ in $A$ of norm $\leqq d$ and define 


$$
\begin{aligned}
\sigma_{m+1}^{\prime}=\sum_{k=1}^{m}(2 d)^{k-1}(2 d+1)^{-k} \mu_{k}+(2 d)^{m}(2 d+1)^{-(m+1)} \mu^{\prime}+ \\
+(2 d)^{m+1}(2 d+1)^{-(m+1)} \varepsilon .
\end{aligned}
$$

That is, $\sigma_{m+1}^{\prime}$ is what $\sigma_{m+1}$ would be if $\mu^{\prime}$ were $\mu_{m+1}$. We rewrite (1):

$$
\sigma_{m+1}^{\prime}=\left(\frac{1}{2 d+1} \mu^{\prime}+\frac{2 d}{2 d+1} \varepsilon\right) \tau_{m},
$$

where

$$
\tau_{m}=\sum_{k=1}^{m}(2 d)^{k-1}(2 d+1)^{-k} \varphi\left(\mu^{\prime}\right) \mu_{k}+(2 d)^{m}(2 d+1)^{-m} \varepsilon .
$$

We can apply Lemma (2.3) with $L$ replaced by $A$ and $c=1$ to infer that

$$
\left\|\varphi\left(\mu^{\prime}\right) \mu_{k}-\mu_{k}\right\| \leqq\left(2+d^{-1}\right)\left\|\mu^{\prime} \mu_{k}-\mu_{k}\right\| .
$$

Consider the condition

$$
\left\|\mu^{\prime} \mu_{k}-\mu_{k}\right\| \text { is sufficiently small for } k=1,2, \ldots, m \text {. }
$$

The set $E$ of invertible elements of $A_{\varepsilon}$ is an open set and the mapping $\chi \rightarrow \chi^{-1}$ is a homeomorphism of $E$ [this is an elementary property of Banach algebras with unit]. Hence if (3) is satisfied, $\left\|\tau_{m}-\sigma_{m}\right\|$ is arbitrarily small, $\tau_{m}^{-1}$ exists, and $\left\|\tau_{m}^{-1}-\sigma_{m}^{-1}\right\|$ is also arbitrarily small. By our hypothesis (2.1.v) on $A$ and $L$, we can satisfy (3) and also the condition

$$
|| \mu^{\prime} \cdot z-z|| \mid \text { is sufficiently small, }
$$

with a single $\mu^{\prime} \in A$ of norm not exceeding $d$.

Using (2) and the preceding remarks, we find

$$
\begin{aligned}
\left\|\left(\sigma_{m+1}^{\prime}\right)^{-1} \cdot z-\sigma_{m}^{-1} \cdot z \mid\right\|= & \left\|\left(\tau_{m}^{-1} \varphi\left(\mu^{\prime}\right)\right) \cdot z-\sigma_{m}^{-1} \cdot z \mid\right\| \\
\leqq & \left\|\left(\tau_{m}^{-1} \varphi\left(\mu^{\prime}\right)\right) \cdot z-\left(\sigma_{m}^{-1} \varphi\left(\mu^{\prime}\right)\right) \cdot z\right\| \|+ \\
& +\|\|\left(\sigma_{m}^{-1} \varphi\left(\mu^{\prime}\right)\right) \cdot z-\sigma_{m}^{-1} \cdot z \mid \| \\
\leqq & c\left\|\tau_{m}^{-1}-\sigma_{m}^{-1}\right\| \cdot\left\|\varphi\left(\mu^{\prime}\right)\right\| \cdot\||\|z\||+ \\
& +c\left\|\sigma_{m}^{-1}\right\| \cdot\left\|\varphi\left(\mu^{\prime}\right) \cdot z-z\right\| \|
\end{aligned}
$$

Notice now that

and

$$
\left\|\varphi\left(\mu^{\prime}\right)\right\| \leqq 2+d^{-1}
$$

$$
|| \varphi\left(\mu^{\prime}\right) \cdot z-z\left|\left\|\leqq\left(2+d^{-1}\right) c||\left|\mu^{\prime} \bullet z-z\right|\right\| \quad\right. \text { (2.3.iii) }
$$

and that ||$z \mid \|$ and $\left\|\sigma_{m}^{-1}\right\|$ are fixed. Thus if (3) and (4) are satisfied by $\mu^{\prime}$, the right side of (5) can be made less than or equal to $2^{-(m+1)} a$. We take any such $\mu^{\prime}$ as $\mu_{m+1}$ and so complete the induction. 
(2.5) Theorem. Let $z$ be any element of $L$, and let $a$ be any positive number. There exist elements $\sigma \in A$ and $y \in L$ with the following properties:

(i) $z=\sigma \cdot y$;

(ii) $y \in(A \bullet z)^{-} \quad$ [closure in $\left.L\right]$;

(iii) ||$|y-z| \| \leqq a$;

(iv) $\|\sigma\| \leqq d$.

Proof. Since the case $z=0$ is trivial, we exclude it in what follows. We use the notation of (2.4). Let $y_{n}=\sigma_{n}^{-1} \bullet z$ for $n=1,2,3, \ldots$, so that $\sigma_{n} \cdot y_{n}=z$. Adding (2.4.ii) from $m+1$ to $m+k$, we have

$$
\left\|\left|y_{m}-y_{m+k}\right|\right\| \leqq 2^{-m} a
$$

for all $m$ and $k$, which proves that $\left(y_{n}\right)_{n=1}^{\infty}$ is a Cauchy sequence in $L$ and so has a limit $y \in L$. Hypothesis (2.1.v) implies that $z \in(A \bullet z)^{-}$, and so plainly $y_{n}$ and $y$ are in $(A \bullet z)^{-}$. Adding (2.4.ii) from 1 to $m$, we see that

$$
\left\|\left|y_{m}-z\right|\right\|<a,
$$

and so $\||y-z|\| \leqq a$. Finally, (2.4.i) shows that $\lim _{n \rightarrow \infty} \sigma_{n}$ exists in $A_{s}$ and is actually the element

$$
\sigma=\sum_{k=1}^{\infty}(2 d)^{k-1}(2 d+1)^{-k} \mu_{k} \in A .
$$

It is clear that $\|\sigma\| \leqq d$. It is also clear that

$$
z=\lim _{n \rightarrow \infty} \sigma_{n} \cdot y_{n}=\left(\lim _{n \rightarrow \infty} \sigma_{n}\right) \cdot\left(\lim _{n \rightarrow \infty} y_{n}\right)=\sigma \cdot y
$$

(2.6) Notes. (a) Suppose that the approximate units of (2.1.v) can always be chosen from a closed subset $C$ of $A$ that contains real positive linear combinations of its own elements. Then it is obvious that $\sigma$ in (2.5) can be taken to be in $C$.

(b) Conditions (2.5.iii) and (2.5.iv) can be changed, if one likes. Replace $\sigma$ by $\|\sigma\|^{-1} \sigma=\sigma^{\prime}$ (note that $\sigma \neq 0$ if $z \neq 0$ ) and $y$ by $\|\sigma\| y=y^{\prime}$. Then we have $z=\sigma^{\prime} \cdot y^{\prime}, y^{\prime} \in(A \bullet z)^{-}$, and $\left\|\sigma^{\prime}\right\|=1$. Condition (2.5.iii) is weakened into

$$
||\left|y^{\prime}-z\right| \| \leqq|||\sigma||-1|(|||z|||+a)+a .
$$

For $d=1$, the inequality ||$z|| \mid c^{-1}(|| z|| \mid+a)^{-1} \leqq\|\sigma\|$ shows that ||$y^{\prime}-z|| \mid$ can be made arbitrarily close to $\left(1-c^{-1}\right)|||z| \|$. 


\section{Applications.}

(3.1) Let $G$ be a locally compact group. Let $\boldsymbol{A}$ be any Banach subalgebra of the algebra $M(G)$ of measures on $G$, and let $L$ be a space $\mathfrak{Q}_{p}(G)$, for $1 \leqq p<\infty$. The operation - of $(2.1)$ is taken to be convolution: $\mu \cdot f$ becomes $\mu * f$, defined as usual by

$$
\mu * f(x)=\int_{G} f\left(y^{-1} x\right) d \mu(y) .
$$

Theorem (20.12) of [2] shows that $\mu * f \in \mathfrak{I}_{p}(G)$ and that (2.1.iv) holds with $c=1$. Properties (2.1.i)-(2.1.iii) are evident.

Theorem (20.15) of [2] shows that $\boldsymbol{A}$ contains an approximate unit for $\mathfrak{Q}_{p}(G)$ if for every neighborhood $U$ of the identity in $G$, there is a $\mu \in A$ such that $\mu\left(U^{\prime}\right)=0, \mu(U)=1$, and $\mu \geqq 0$. In fact for $f \in \mathfrak{R}_{p}(G)$ and $a>0$, there is a $U$ such that

$$
\|\mu * f-f\|_{p}<a
$$

for all $\mu \in M(G)$ such that $\mu\left(U^{\prime}\right)=0, \mu(U)=1$, and $\mu \geqq 0$. Not all Banach subalgebras of $\boldsymbol{M}(G)$ containing $\mu$ 's with arbitrarily small supports admit approximate left units for themselves, however. To apply (2.5) an approximate left unit for $\boldsymbol{A}$ itself is of course needed.

(3.2) Theorem. Let $\boldsymbol{A}$ be a Banach subalgebra of $\boldsymbol{M}(G)$ with the following property. For every $\left\{\mu_{1}, \ldots, \mu_{m}\right\} \subset \boldsymbol{A}$, every neighborhood $U$ of the identity in $G$, and $a>0$, there is a nonnegative measure $v$ in $A$ such that $v(U)=1$ and $v\left(U^{\prime}\right)=0$ and

$$
\left\|v * \mu_{j}-\mu_{j}\right\|<a \quad \text { for } \quad j \in\{1,2, \ldots, m\} .
$$

Let $f$ be any function in $\mathfrak{R}_{p}(G), 1 \leqq p<\infty$, and $b$ any positive number. Then there exist a nonnegative measure $\sigma$ of norm 1 in $\boldsymbol{A}$ and a function $g \in \mathfrak{Q}_{p}(G)$ such that:

(i) $f=\sigma * g$;

(ii) $g \in(\boldsymbol{A} * f)^{-}$;

(iii) $\|g-f\|_{p} \leqq b$;

(iv) $g$ is real-valued if $f$ is.

Proof. Apply (2.5), taking note of (2.6.a).

(3.3) Corollary. For every $f$ in $\mathfrak{R}_{p}(G), 1 \leqq p<\infty$, neighborhood $U$ of the identity in $G$, and $a>0$, there exist functions $g \in \mathfrak{R}_{p}(G)$ and $h \in \mathbb{R}_{1}^{+}(G)$ such that:

(i) $f=h * g$;

(ii) $g \in\left(\mathfrak{L}_{1}(G) * h\right)^{-}$; 
(iii) $\|g-f\|_{p}<a$;

(iv) $\int_{G} h d \lambda=1$ and $h\left(U^{\prime}\right) \subset\{0\}$;

(v) $g$ is real-valued if $f$ is.

(3.4) Remarks. Crudely speaking, (3.3) asserts that $\mathfrak{L}_{1}(G) * \mathfrak{L}_{p}(G)=$ $\mathfrak{Q}_{p}(G)$. Theorem (2.5) is applicable to a number of function spaces other than $\mathfrak{L}_{p}(G)$. For example, let $\mathfrak{E}_{r u}(G)$ be the bounded, right uniformly continuous, complex-valued functions on $G$. Then the analogue of (3.3) holds with $\mathfrak{R}_{p}(G)$ replaced by $\mathfrak{C}_{r u}(G)$ and $\|\cdot\|_{p}$ by the uniform norm $\|\cdot\|_{u}$. Thus too we have $\mathfrak{L}_{1}(G) * \mathfrak{C}_{r u}(G)=\mathfrak{C}_{r u}(G)$.

A slightly different situation arises for convolutions on the right, since the modular function for $G$ must enter. With no wish to flog a dead horse, we state the results briefly.

(3.5) Theorem. Suppose that $1<p<\infty$. Let $\mathfrak{Q}_{1, p^{\prime}}(G)$ denote the space of all Borel measurable, complex-valued functions on $G$ for which

(i) $\|h\|_{1, p^{\prime}}=\int_{G}|h(x)| \Delta(x)^{-1 / p^{\prime}} d \lambda(x)<\infty$,

where $p^{\prime}=p /(p-1)$. The space $\mathfrak{L}_{1, p^{\prime}}(G)$ is a Banach algebra under convolution $\left[h * k(x)=\int_{G} h(x y) k\left(y^{-1}\right) d \lambda(y)\right]$. Let $f$ be any function in $\mathfrak{Q}_{p}(G), U$ any neighborhood of the identity in $G$, and a any positive number. There exist functions $g \in \mathfrak{L}_{p}(G)$ and $h \in \mathfrak{R}_{1, p^{\prime}}^{+}(G)$ such that:

(ii) $f=g * h$;

(iii) $g \in\left(h * \mathfrak{R}_{\mathbf{1}, p^{\prime}}(G)\right)^{-}$;

(iv) $\|g-f\|_{p}<a$;

(v) $\|h\|_{1, p^{\prime}}=1$ and $h\left(U^{\prime}\right) \subset\{0\}$;

(vi) $g$ is real-valued if $f$ is.

Proof. Theorem (2.5) has an obvious "right-handed" version using approximate right units for $A$ and $L$ and the condition $(\mu \nu) \bullet x=\nu \cdot(\mu \cdot x)$ instead of (2.1.iii). Theorem (20.13) of [2] shows that the convolution mapping

$$
(f, g) \rightarrow f * g
$$

for $f \in \mathfrak{Q}_{p}(G)$ and $g \in \mathfrak{R}_{1, p^{\prime}}(G)$ satisfies (2.1.i), (2.1.ii), and (2.1.iv). Theorem (20.15) of [2] shows that approximate right units exist.

(3.6) For convolutions $g * h$ where $g$ is merely bounded, we have yet another result. Let $\mathfrak{C}_{l u}(G)$ be the space of bounded, left uniformly continuous, complex-valued functions on $G$. Let $\mathfrak{R}_{1}^{\star}(G)$ be the space of all functions $h$ on $G$ for which $h^{\star}\left[h^{\star}(x)=h\left(x^{-1}\right)\right]$ is in $\mathfrak{L}_{1}(G)$. Then the ana- 
logue of Theorem (3.5) holds with $\mathfrak{R}_{p}(G)$ replaced by $\mathfrak{C}_{l u}(G), \mathfrak{L}_{1, p^{\prime}}(G)$ by $\mathfrak{Q}_{1}(G)$, and $\|\cdot\|_{p}$ by $\|\cdot\|_{u}$.

(3.7) FAILURe OF THe theOREM for $\mathfrak{Q}_{p}(G) * \mathfrak{Q}_{q}(G)$. For $p>1$ and $q>1$, the functions in $\mathfrak{Q}_{p}(G) * \mathfrak{Q}_{q}(G)$ may be a small subspace of both $\mathfrak{Q}_{p}(G)$ and $\mathfrak{L}_{q}(G)$. For example, let $G$ be a compact group and $p=q=2$. Then $\mathfrak{L}_{2}(G) * \mathfrak{L}_{2}(G)$ is the space of all complex linear combinations of continuous positive-definite function on $G$, a space that can be characterized in a quite concrete way, as M. G. Krein has shown, ([3], Theorem 12). In the Abelian case, this space is just the space of functions on $G$ with absolutely convergent Fourier series. Naturally $\mathfrak{L}_{2}(G)$ is a Banach algebra under convolution ( $G$ is compact) and it has approximate left and right units ([2], (20.15)). These approximate units are not bounded in the $\mathfrak{L}_{\mathbf{2}}$ norm, however; if they were, then Theorem (2.5) would apply and $\mathfrak{L}_{2}(G) * \mathfrak{L}_{2}(G)$ would be $\mathfrak{L}_{2}(G)$. Notice nonetheless that the identity

$$
\lim _{U \rightarrow\{e\}}\left\|\varphi_{U} * f-f\right\|_{2}=0,
$$

which holds for each $f \in \mathfrak{I}_{2}(G)$, allows us to apply the uniform boundedness principle. The norms of the operators $f \rightarrow \varphi_{U} * f$ on $\mathfrak{L}_{2}(G)$ have a common bound, but the number $\left\|\varphi_{U}\right\|_{2}$ (which may or may not be finite) cannot possibly be bounded.

(3.8) Open PRoblems. For $1<p<2$, an inequality

(i) $\|f * g\|_{2} \leqq A_{p}\|f\|_{p}\|g\|_{2}$

for $f \in \mathfrak{R}_{p}(G)$ and $g \in \mathfrak{L}_{2}(G)$ may or may not obtain. The relation (i) with $A_{p}=1$ is simple to check if $G$ is compact. It has been established for $G=\mathfrak{S} \mathfrak{Q}(2, R)$ by Kunze and Stein ([4, Theorem 9]). No inequality (i) holds for $G=R$, as Kunze and Stein also show $([4$, p. 61]). For groups $G$ such that (i) holds, it would be interesting to know what $\mathfrak{Q}_{p}(G) * \mathfrak{L}_{2}(G)$ is.

Young's inequality $([2,(20.18)])$ is

(ii) $\mathfrak{L}_{p}(G) * \mathfrak{L}_{q}^{\star}(G) \subset \mathfrak{R}_{r}(G)$,

where $1<p<\infty, 1<q<\infty$, and $1 / r=1 / p+1 / q-1>0$. This inequality at once raises the problem of determining exactly what the set $\mathfrak{L}_{p}(G) * \mathfrak{L}_{q} \star(G)$ is.

\section{LITERATURE}

1. Paul J. Cohen, Factorization in group algebras, Duke Math. J. 26 (1959), 199-205.

2. Edwin Hewitt and Kenneth A. Ross, Abstract harmonic analysis I, Berlin · Göttingen • Heidelberg, 1963. 
3. M. G. Kreĭn, Hermitian-positive kernels on homogeneous spaces I, II, Ukrain. Mat. Ž. 1 (1949), 64-98; 2 (1950), 10-59.

4. R. A. Kunze and E. M. Stein, Uniformly bounded representations and harmonic analysis of the $2 \times 2$ real unimodular group, Amer. J. Math. 82 (1960), 1-62.

5. Nicholas Th. Varopoulos, Sur les formes positives d'une algebre de Banach, C. R. Acad. Sci. Paris 258 (1964), 2465-2467.

UNIVERSITY OF WASHINGTON,

SEATTLE, WASH., U.S.A. 